\title{
GMR
}

\section{Molecular characterization, chromosome mapping, and expression profile of porcine CDC16}

\author{
D. Xu, J. Jiang and H. Ma \\ College of Animal Science and Technology, Hunan Agricultural University, \\ Changsha, People's Republic of China \\ Corresponding author: H. Ma \\ E-mail: mahaiming2000@163.com \\ Genet. Mol. Res. 14 (4): 12811-12819 (2015) \\ Received May 18, 2015 \\ Accepted August 19, 2015 \\ Published October 19, 2015 \\ DOI http://dx.doi.org/10.4238/2015.October.19.25
}

\begin{abstract}
Cell division cycle16 (CDC16) is a core component among the eight protein subunits of the anaphase-promoting complex (APC). APC is a cyclin degradation system that governs the exit of cells from mitosis. Not much information is available for $C D C 16$ in pig. In this study, a 2284-bp cDNA of porcine CDC16 was obtained by rapid amplification of CDNA ends (RACE). Porcine CDC16 was assigned to SSC11 q11-17, and was determined to be significantly linked with SW1452 by using somatic cell hybrid panel and radiation hybrid panel. One novel A/G SNP anchored in intron 7 of the gene was genotyped by restriction enzyme polymerase chain reaction (PCR)-restriction fragment length polymorphism-Csp6I. In five pig breeds, Shaziling, Taoyuan, Duroc, Landrace, and Yorkshire, the $A$ allele frequency was dominant. Quantitative PCR revealed that porcine CDC16 was expressed in ten selected tissues of 25-day-old Shaziling and Yorkshire piglets, and that the mRNA expression of $C D C 16$ in longissimus dorsi muscle of Shaziling was higher than that of Yorkshire. Expression levels of $C D C 16$ were highest in longissimus dorsi muscle followed by that in pancreas. CDC16 protein was detected in longissimus dorsi muscle of 25-day-old Shaziling and Yorkshire piglets by immunohistochemistry with
\end{abstract}


abundant protein expression index $(P>0.05)$. This study provides an insight into the role of porcine $C D C 16$ in the formation of meat.

Key words: Pig; $C D C 16$; Chromosomal localization; Expression profile; Immunohistochemistry

\section{INTRODUCTION}

Cell division cycle 16 is a protein encoded by the gene $C D C 16$ in humans (Tugendreich et al., 1995). This protein is one of the components of anaphase-promoting complex (APC) which is composed of eight proteins, namely, BimE (APC1), APC2, CDC27 (APC3), APC4, APC5, CDC16 (APC6), APC7, and CDC23 (APC8), and it functions as a protein ubiquitin ligase (Yu et al., 1998). APC is a cyclin degradation system that governs the exit of cells from mitosis. Each component protein of the APC is highly conserved among eukaryotic organisms. CDC16 contains a tetratricopeptide repeat (TPR), a protein domain that may be involved in protein-protein interaction (Ollendorff and Donoghue, 1997; Kallio et al., 1998; Vodermaier et al., 2003). CDC16 has been demonstrated to interact with CDC27 and CDC20 (Gmachl et al., 2000; Nilsson et al., 2008).

A recent report suggested that $\mathrm{CDC} 16$ plays an important role in differentiation of epithelial cells in the hair follicle (Ollendorff and Donoghue, 1997). Immunofluorescence analysis revealed that, during mitosis, CDC16 accumulated in the central body including the spindle (Tugendreich et al., 1995). In an RNA interference (RNAi) screen utilizing endoribonuclease-prepared short interfering (esi) RNA library, CDC16 was demonstrated to be involved in cell division (Kittler et al., 2004). By fluorescence in situ hybridization, human CDC16 was found to be localized on HSA13q34 (Kallio et al., 1998; Kallio et al., 2002). The gene was demonstrated to be located on mouse (Mus musculus) chromosome 8A1.1-1.2 (Skarnes et al., 2011) and on rat (Rattus norvegicus) chromosome 16q12.5 (Steen et al., 1999). The aim of the present study was to gain an insight into the function of porcine CDC16 during processing of the meat.

\section{MATERIAL AND METHODS}

\section{Isolation of cDNA}

Total RNA was isolated from longissimus dorsi muscle of one, 25-day-old, weaned Shaziling piglet using Trizol reagent (Invitrogen, Karlsruhe, Germany). The isolated RNA was reverse-transcribed using Moloney Murine Leukemia Virus reverse transcriptase (Promega, USA), according to the manufacturer instructions; the RT-reaction product was used as a template for further analysis. Several pig ESTs were initially identified using the cDNA sequence of human CDC16 mRNA (NM_004661) by a BLASTN search against the GenBank EST databases by Sequence alignment. The porcine ESTs sharing at least $80 \%$ identity to the corresponding human mRNA were selected for designing gene-specific primers (Table 1). The PCR products were purified with a Gel Extraction Mini Kit (Qiagen, Hamburg, Germany), cloned into PGEMT plasmid (Promega, USA), sequenced by using random amplification of CDNA ends (RACE) methods. 


\begin{tabular}{|c|c|c|c|c|}
\hline Primer's purpose & Primer name & Primer sequence $\left(5^{\prime}-3^{\prime}\right)$ & $\operatorname{Tm}\left({ }^{\circ} \mathrm{C}\right)$ & Product size $(b p)$ \\
\hline \multirow[t]{4}{*}{ Cloning } & $\mathrm{RC} 11-1 \mathrm{~F}$ & GGAAATGTCACAGTCCTCAATA & 58 & 1086 \\
\hline & $\mathrm{RC} 11-1 \mathrm{R}$ & CAGAAAATGTATCATCTCGCCT & & \\
\hline & $\mathrm{RC} 11-2 \mathrm{~F}$ & TGAGGTGACAGTTGACAAATGGGA & 58 & 816 \\
\hline & $\mathrm{RC} 11-2 \mathrm{R}$ & CCATAGGCTACATCCATAGTCTGA & & \\
\hline \multirow[t]{2}{*}{ Gene mapping } & $\mathrm{M}-\mathrm{F}$ & GCGCAGTATC ATAGAGCAGC & 58 & 743 \\
\hline & $M-R$ & GGTAGCGACA CGCTTCATAC & & \\
\hline \multirow[t]{2}{*}{ Polymorphism } & Genomic-F & GACACGGCAACATGCTAG & 50.5 & 696 \\
\hline & Genomic-R & TTGGGATGGAAGAAGACC & & \\
\hline \multirow[t]{2}{*}{ Expression profile } & CDC16-F & CCGAGACACTGAATTTTG & 60 & 51 \\
\hline & CDC16-R & ACTGAGGTTAGTCTGCTAM & & \\
\hline \multirow[t]{2}{*}{ Internal control } & GAPDH-F & ATTTGGCTACAGCAACAGGGT & 59 & 172 \\
\hline & GAPDH-R & AAGTCAGGAGATGCTCGGTGT & & \\
\hline
\end{tabular}

\section{Mapping of porcine CDC16}

A somatic cell hybrid panel (SCHP) was used for chromosomal mapping and an INRAUniversity of Minnesota porcine radiation hybrid (IMpRH) was employed for ascertaining the precise location of porcine $C D C 16$. Primers, corresponding to exon 4 and 5 and intron 4 of pig $C D C 16$, were designed (Table 1). A 743 bp PCR product for CDC16 was used to type SCHP and IMpRH panel (Yerle et al., 1999, 2001). The PCR products were visualized on $2 \%$ agarose gel stained with ethidium bromide. The PCR results were statistically analyzed using SCHP and IMpRH mapping tools, accessible through the websites, http://www2. toulouse. inra.fr/lgc/pig/pcr/pcr.htm and http:// www. imprh. toulouse. inra.fr, respectively (Milan et al., 2000).

\section{Novel SNP identification using PCR-RFLP test}

Samples were collected from eight breeds of pig viz. Duroc $(N=62)$, Landrace $(N=45)$, Yorkshire $(N=443)$, Shaziling $(N=62)$, Daweizi $(N=63)$, Taoyuan $(N=70)$, Ningxiang $(N=67)$, and Wuzhishan ( $\mathrm{N}=54$ ) provided by Hunan Xinwufeng Breeding Company in Pig (Xiangtan City, Hunan Province, China) and Resource Field of Chinese native pig. Genomic DNA extracted using EasyPure Genomic DNA Kit (Transgen Biotech, Beijing, China) was amplified and sequenced directly for SNP identification. For genotyping, the PCR was performed in a $20 \mu \mathrm{L}$ mixture consisting of $100 \mathrm{ng}$ genomic DNA, $25 \mathrm{pmol}$ primers, $75 \mu \mathrm{M}$ dNTPs, $1.5 \mathrm{mM} \mathrm{MgCl}$, and $0.5 \mathrm{U}$ Taq DNA polymerase. The PCR conditions were: $94^{\circ} \mathrm{C}$ for $5 \mathrm{~min}$, followed by 35 cycles of $94^{\circ} \mathrm{C}$ for $30 \mathrm{~s}$, $50.5^{\circ} \mathrm{C}$ for $30 \mathrm{~s}$, and $72^{\circ} \mathrm{C}$ for $30 \mathrm{~s}$, and a final extension at $72^{\circ} \mathrm{C}$ for $10 \mathrm{~min}$. PCR product $(4 \mu \mathrm{L})$ was digested overnight with $3 \cup \mathrm{Csp} 6 \mathrm{l}$ (Thermo Fisher Scientific, USA) at $37^{\circ} \mathrm{C}$, electrophoresed on a $2.0 \%$ agarose gel and visualized by staining with ethidium bromide.

\section{Expression profile of $C D C 16$}

Total RNA was isolated from heart, liver, spleen, lung, kidney, intestine, cecum, pancreas, crureus, and longissimus dorsi muscle of three, 25-day-old, weaned Yorkshire piglets and three weaned Shaziling piglets of the same age. These two pig breeds are full-sib. The cDNA from the above ten tissues obtained after reverse transcription was used as template in quantitative PCR, using SYBR Green I method (Livak and Schmittgen, 2001). The reaction mixture $(25 \mu \mathrm{L})$ contained $2 \mu \mathrm{L}$ RT-PCR product, $12.5 \mu \mathrm{L} 2 \mathrm{X}$ SYBR Premix Ex Taq II, $0.5 \mu \mathrm{L}$ each CDC16-F and CDC16-R 
primers, and $9.5 \mu \mathrm{L}$ RNase-free double-distilled $\mathrm{H}_{2} \mathrm{O}$. The reaction conditions were: $95^{\circ} \mathrm{C}$ for 30 $\mathrm{s}$ followed by 40 cycles of $95^{\circ} \mathrm{C}$ for $50 \mathrm{~s}$ and $60^{\circ} \mathrm{C}$ for $30 \mathrm{~s}$ and $72^{\circ} \mathrm{C}$ for $30 \mathrm{~s}$. Average results from three technical replicates per test sample were used for analysis. The $2^{-(\Delta \Delta C t)}$ method (Livak and Schmittgen, 2001) was used to calculate the relative expression of the target gene. GAPDH (glyceraldehydes-3-phosphate dehydrogenase) gene was used as an internal control. The cecum sample from Yorkshire was defined as the reference, and internal control primers were designed according to the pig mRNA (Table 1).

\section{Immunohistochemical experiment}

For immunohistochemical experiments (Fregly and Graybiel, 1968; Nishiwaki et al., 2000), the longissimus dorsi muscle tissue was embedded in paraffin after fixing in 4\% paraformaldehyde. Sections ( $4 \mu \mathrm{m}$ thick), were dehydrated by passing through an alcohol series. They were incubated at $4^{\circ} \mathrm{C}$ overnight with bs-7832R monoclonal antibody (1:100 dilution) specific for CDC16 protein and washed 3 times with phosphate buffered saline (PBS) for $5 \mathrm{~min}$. Thereafter, the sections were stained with hematoxylin and eosin, as described previously (Cerri and Sasso-Cerri, 2003). Meat tissue micrographs were taken at a magnification of 400X with an Axio Imager Z1 (ZEISS, Germany).

\section{RESULTS}

\section{Cloning and characterization of CDC16 cDNA}

The 2284 bp porcine CDC16 cDNA (GenBank accession No. KC795824) was obtained by RACE. It contained an 1863 bp open reading frame (ORF) flanked by a 38 bp $5^{\prime}$-untranslated region (UTR) and a 383 bp 3'-UTR, which encoded the deduced 620 amino acids. The molecular weight of CDC16 was about $188.3 \mathrm{kDa}$, and its calculated isoelectric point $(\mathrm{PI})$ was 4.92. Sequence alignment showed that the complete cDNA sequence shared 92 and $83 \%$ identity with human (NM_003903.3) and mouse (NM_027276) sequences, respectively.

\section{Map assignment of porcine CDC16}

By the somatic cell hybrid panel, CDC16 was mapped to Sus scrofa chromosome (SSC) $11 q 11-q 17$, with the probability of regional localization being 0.9998 , correlation 0.8563 and error risk $<0.1 \%$. Further mapping was conducted using the radiation hybrid panel IMpRH. The statistical analysis revealed that $C D C 16$ was significantly linked to microsatellite SW1452 with a distance of $56 \mathrm{cR}$ and LOD score of 16.08 on chromosome 11 (Figure 1). Human CDC16 was assigned to HSA 13 (Johansson et al., 1995). Our results were consistent with previous results because human chromosome 13 is homologous with porcine chromosome 11 [http://www2.toulouse.inra.fr/lgc/pig/ compare/SSC.htm; Chevalet et al. (1997)].

\section{Polymorphism detection}

A 696 bp genomic fragment spanning the intron 7 of CDC16 was amplified and one novel SNP was genotyped with PCR-RFLP-Csp6I (696 bp for allele $A$, and 570 and 126 bp for allele G; Figure 2). The results indicated that the frequency of allele $A$ was greater than 0.500 , in Yorkshire, Landrace, Duroc, Taoyuan, and Shaziling pigs (Table 2). In Daweizi, Ningxiang, and Wuzhishan 
pig, the frequency of allele $G$ was lesser than 0.500 , and in the three European breeds, Duroc, Landrace and Yorkshire, alleles $A$ was dominant. In Taoyuan pig, the frequency of allele $A$ was highest (0.7570).

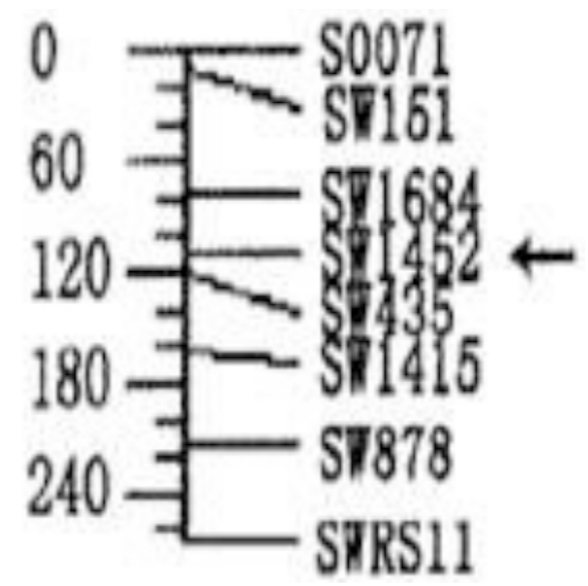

Figure 1. Radiation hybrid mapping of pig CDC16 onto SSC11.

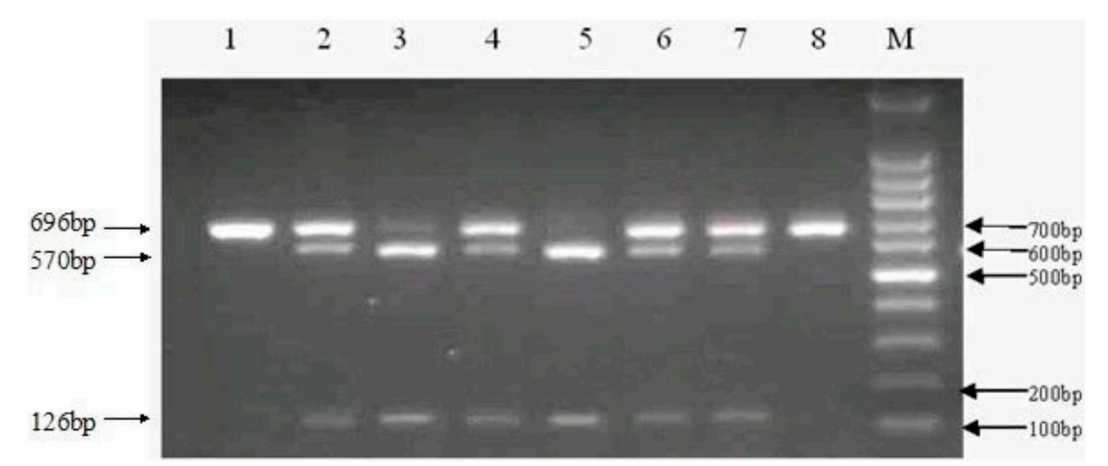

Figure 2. Novel single nucleotide polymorphism (SNP) detected in CDC16. Polymerase chain reaction (PCR) products were digested with Csp6l restriction enzyme to distinguish different alleles. The picture shows electrophoresis of different genotype samples on a $2 \%$ agarose gel (lanes 1-8). M: 100 bp DNA ladder.

Table 2. Frequency of allele and genotype of CDC16 about PCR-RFLP-Csp6 I among different pig breeds.

\begin{tabular}{|c|c|c|c|c|c|c|c|c|c|c|}
\hline \multirow[t]{2}{*}{ Breed } & \multirow[t]{2}{*}{ Sample size } & \multicolumn{3}{|c|}{ Genotype } & \multicolumn{3}{|c|}{ Frequency of genotype } & \multicolumn{2}{|c|}{ Frequency of allele } & \multirow[t]{2}{*}{$x^{2}$} \\
\hline & & AA & AG & GG & AA & AG & GG & A & G & \\
\hline Ningxiang & 67 & 13 & 41 & 13 & 0.1912 & 0.6029 & 0.2059 & 0.4926 & 0.5074 & 2.8897 \\
\hline Daweizi & 63 & 2 & 38 & 23 & 0.0317 & 0.6032 & 0.3651 & 0.3333 & 0.6667 & 8.0357 \\
\hline Shaziling & 62 & 17 & 31 & 14 & 0.2742 & 0.5000 & 0.2258 & 0.5242 & 0.4758 & 0.0003 \\
\hline Taoyuan & 70 & 38 & 30 & 2 & 0.5429 & 0.4286 & 0.0285 & 0.7570 & 0.2430 & 1.9143 \\
\hline Wuzhishan & 54 & 5 & 34 & 15 & 0.0926 & 0.6296 & 0.2778 & 0.4074 & 0.5926 & 4.9897 \\
\hline Yorkshire & 443 & 231 & 185 & 27 & 0.5215 & 0.4176 & 0.0609 & 0.7302 & 0.2698 & 1.5945 \\
\hline Landrace & 45 & 24 & 17 & 4 & 0.5333 & 0.3778 & 0.0889 & 0.7222 & 0.2778 & 0.1538 \\
\hline Duroc & 62 & 35 & 18 & 9 & 0.5646 & 0.2903 & 0.1451 & 0.7097 & 0.2903 & 5.4122 \\
\hline
\end{tabular}




\section{Expression profile analysis}

Quantitative PCR showed that the porcine CDC16 expressed in ten tissues of 25-day-old Yorkshire and Shaziling pigs (Figure 3). The constitutively expressed GAPDH was used as an endogenous reference in the analysis. The level of the $C D C 16$ expression in intestine of Yorkshire pig was defined as 100 and the mRNA expression level in different tissues was quantified relative to it. CDC16 mRNA expressed in all the ten tissues, including intestine, caecum, heart, liver, kidney, lung, spleen, pancreas, crureus, and longissimus dorsi muscle. Highest expression was observed in the longissimus dorsi and the lowest in the lung and kidney of the Shaziling and Yorkshire pigs, respectively $(P<0.01)$. CDC16 expression in the cecum and heart of Yorkshire and Shaziling pigs was significantly different $(P<0.01)$. CDC16 was widely expressed in the assessed organs of Yorkshire and Shaziling pigs. It may be related to porcine cytokines and hormones involved in intracellular signal transduction.

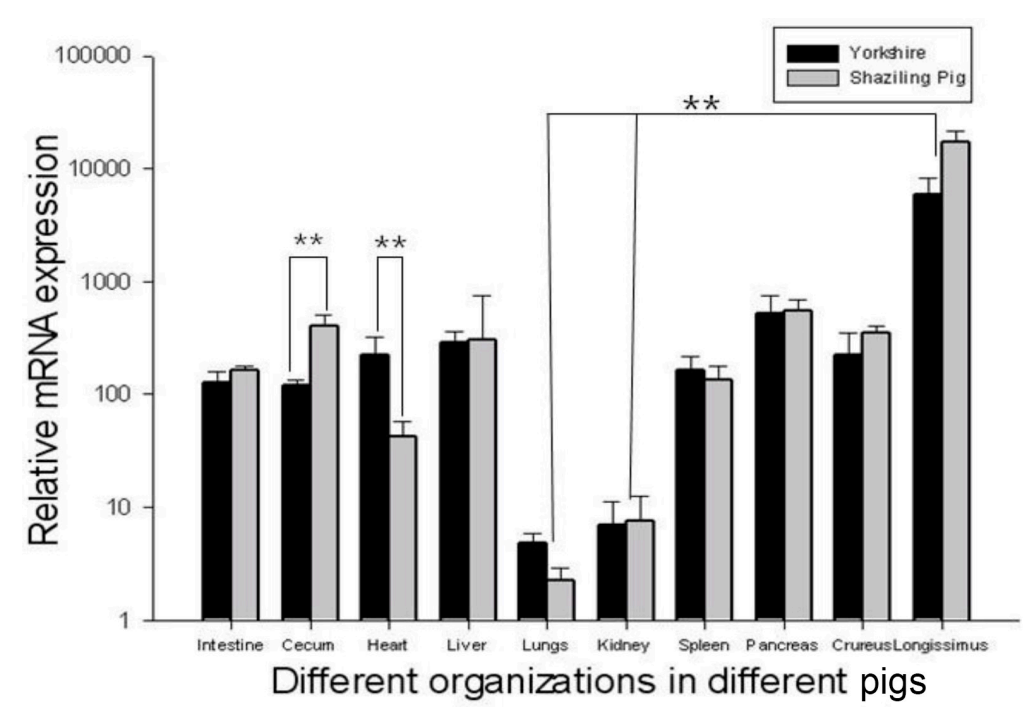

Figure 3. CDC16 expression in ten tissues of 25-day-old Yorkshire and Shaziling pigs evaluated by qRTPCR. The expression level was normalized to GAPDH and measured by $2-\Delta \Delta c t$. Results from three independent replicates in different tissues were averaged. **Significant difference between the two breeds $(P<0.01)$.

\section{Histochemistry of CDC16 protein in longissimus dorsi}

In this study, CDC16 protein was confirmed as a membrane protein and was detected in longissimus dorsi muscle tissue of Shaziling and Yorkshire piglets by immunohistochemistry which revealed abundant protein expression index $(P E I)(P>0.05)$. It is interesting that $q P C R$ results for $C D C 16$ expression were contrary to that of histochemistry. This signified that the expression of $C D C 16$ was different from that of its protein in longissimus dorsi (Figure 4). In Yorkshire piglets, average optical density was 0.34 , whereas in Shaziling, it was 0.37 . According to the statistical analysis, no significant difference was observed between the two average optical densities $(P>0.05)$. 


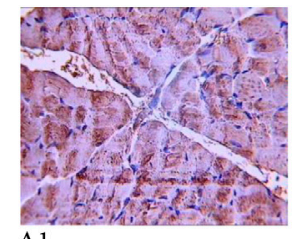

Al

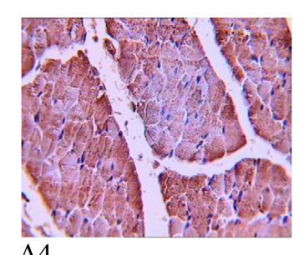

A4

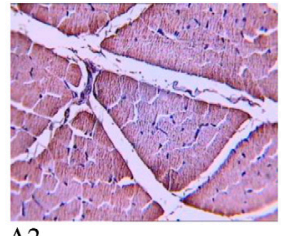

A2

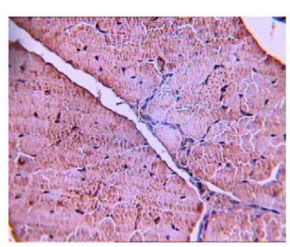

A5

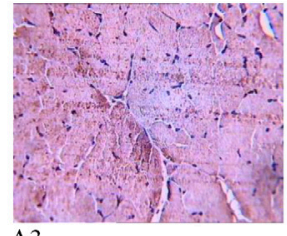

A3

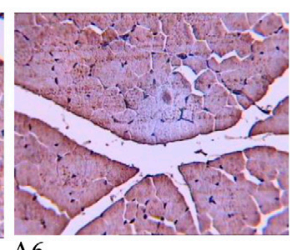

Figure 4. Expression of $\mathrm{CDC} 16$ protein in longissimus dorsi muscle tissue. A1-A3 indicate the expression in Yorkshire piglets. A4-A6 indicate expression in Shaziling piglets. Blue specks in the pictures denote the location of the CDC16 protein. All pictures were taken at a magnification of $400 \mathrm{X}$.

\section{DISCUSSION}

Human $C D C 16$ is made up of 18 exons and 17 introns and its $5^{\prime}-$ UTR is $186 \mathrm{bp}, 3^{\prime}-$ UTR is $232 \mathrm{bp}$, full length of the gene is $43.8 \mathrm{~kb}$, and full-length of cDNA is $2461 \mathrm{bp}$ (Tugendreich et al., 1995). Mouse CDC16 consists of 7 exons and 6 introns with 110 bp 5'-UTR, 299 bp 3'-UTR, gene length $12676 \mathrm{bp}, \mathrm{cDNA}$ length $2253 \mathrm{bp}$, and ORF length $1763 \mathrm{bp}$. In this study, we performed RACE-PCR to obtain a 2284 bp full-length porcine CDC16 cDNA having 1863 bp ORF flanked by a 38 bp 5'-UTR and 383 bp 3'-UTR that putatively encoded for 675 amino acids.

Our report indicates that allele $A$ was predominant in the three European and Chinese indigenous breeds of Taoyuan pig, and the highest $A$ gene frequency was 0.7570 in Taoyuan pig, whereas the highest $G$ gene frequency was 0.6667 in Daweizi pig. The $A A$ genotype frequency of Chinese breeds was superior to that of European breeds, whereas in native pigs, heterozygous $A G$ genotype frequency was superior, except for the Taoyuan pigs.

The relative mRNA expression of CDC16 varied in different tissues. CDC16 mRNA expression was observed in the intestine, caecum, heart, liver, kidney, lung, spleen, pancreas, crureus, and longissimus dorsi, and significant differences existed among the different organs $(P$ $<0.05$ ). Highest expression was observed in the longissimus dorsi and the lowest was in the lung and kidneys of the Shaziling and Yorkshire pigs. Rest of the organs, especially the cecum and heart of Yorkshire and Shaziling pigs has high expression that was significant different $(P<$ 0.01). $C D C 16$ in Yorkshire and Shaziling pigs was widely distributed in tissues, and it could be related to porcine cytokines and hormones in the body, that function during intracellular signal transduction. CDC16 has been reported to play a key role in cell division. In the present study, CDC16 mRNA expression was estimated in ten tissues of Shaziling and Yorkshire. Its expression in longissimus dorsi was higher than in other tissues, and the lowest expression was in lungs and kidney, indicating that $C D C 16$ could be associated with muscle growth, playing an important regulatory role during muscle development in pigs. The cecum and heart showed highly significant differences between Yorkshire and Shaziling piglets, which may be caused by the discrepancy in the initial development in the different breeds. It has an important significance to account for the genetic differences between native Chinese and European pigs. 
Immunohistochemistry was carried out to quantitatively characterize the expression of proteins related to the meat quality. In histological examination, muscle phenotype variation was apparent at the 25-day stage. Moreover, there is fiber type-transition and muscle maturation process from the birth till the age of two months (Lefaucheur and Vigneron, 1986). Thus, CDC16 might contribute to the formation of muscle fibers in Yorkshire and Shaziling pigs.

\section{CONCLUSIONS}

In summary, the complete cDNA of pig CDC16 was isolated and characterized and one novel SNP in intron 7 was identified in CDC16. It was closely linked with microsatellite SW1452 of the SSC11. The spatial expression profiles of pig CDC16 mRNA displayed its abundance in longissimus dorsi with significant differences between Shaziling and Yorkshire pig. However, the expression of CDC16 protein in longissimus dorsi was not significantly different between these two breeds. This suggests that the expression of $C D C 16$ was different at the level of transcription and translation in longissimus dorsi.

\section{Conflicts of interest}

The authors declare no conflict of interest

\section{ACKNOWLEDGMENTS}

Research supported by the National High Science and Technology Foundation of China (Grant \#2011AA100304), Provincial Natural Science Foundation of Hunan (Grant \#13JJ1021) and Key Foundation of Education Department in Hunan province (Grant \#12A060).

\section{REFERENCES}

Cerri P and Sasso-Cerri E. (2003). Staining methods applied to glycol methacrylate embedded tissue sections. Micron. 34: 365-372.

Chevalet C, Gouzy J and SanCristobal-Gaudy M. (1997). Regional assignment of genetic markers using a somatic cell hybrid panel: a WWW interactive program available for the pig genome. Computer applications in the biosciences: CABIOS 13: 69-73.

Fregly AR and Graybiel A (1968). An ataxia test battery not requiring rails. Aerospace Med. 39: 277-282.

Gmachl M, Gieffers C, Podtelejnikov AV, Mann M, et al. (2000). The RING-H2 finger protein APC11 and the E2 enzyme UBC4 are sufficient to ubiquitinate substrates of the anaphase-promoting complex. Proc. Nat. Acad. Sci. 97: 8973-8978.

Johansson M, Ellegren H and Andersson L. (1995). Comparative mapping reveals extensive linkage conservation-but with gene order rearrangements-between the pig and the human genomes. Genomics 25: 682-690.

Kallio M, Weinstein J, Daum JR, Daniel J. Burke, et al. (1998). Mammalian p55CDC mediates association of the spindle checkpoint protein Mad2 with the cyclosome/anaphase-promoting complex, and is involved in regulating anaphase onset and late mitotic events. The J. Cell Biol. 141: 1393-1406.

Kallio MJ, Beardmore VA, Weinstein J and Gary J. Gorbsky (2002). Rapid microtubule-independent dynamics of Cdc20 at kinetochores and centrosomes in mammalian cells. The J. Cell Biol. 158: 841-847.

Kittler R, Putz G, Pelletier L, Poser I, et al. (2004). An endoribonuclease-prepared siRNA screen in human cells identifies genes essential for cell division. Nature 432: 1036-1040.

Lefaucheur L and Vigneron P. (1986). Post-natal changes in some histochemical and enzymatic characteristics of three pig muscles. Meat. Sci. 16: 199-216.

Livak KJ and Schmittgen TD. (2001). Analysis of relative gene expression data using real-time quantitative PCR and the $2^{-\Delta \Delta C T}$ method. Methods 25: 402-408.

Milan D, Hawken R, Cabau C, Leroux S, et al. (2000). IMpRH server: an RH mapping server available on the Web. Bioinformatics 16: 558-559. 
Nilsson J, Yekezare M, Minshull J, Pines J, et al. (2008). The APC/C maintains the spindle assembly checkpoint by targeting Cdc20 for destruction. Nat. Cell Biol. 10: 1411-1420.

Nishiwaki Y, Takebayashi T, Imai A, Yamamoto M. et al. (2000). Difference by instructional set in stabilometry. J. Vest. Res. 10: $157-161$.

Ollendorff $\vee$ and Donoghue DJ. (1997). The serine/threonine phosphatase PP5 interacts with CDC16 and CDC27, two tetratricopeptide repeat-containing subunits of the anaphase-promoting complex. J. Biol. Chemistry 272: 32011-32018.

Skarnes WC, Rosen B, West AP, Koutsourakis M, et al. (2011). A conditional knockout resource for the genome-wide study of mouse gene function. Nature 474: 337-342.

Steen RG, Kwitek-Black AE, Glenn C, Gullings-Handley J, et al. (1999). A high-density integrated genetic linkage and radiation hybrid map of the laboratory rat. Genome Res. 9: AP1-AP8.

Tugendreich S, Tomkiel J, Earnshaw W, Hieter P, et al. (1995). CDC27Hs colocalizes with CDC16Hs to the centrosome and mitotic spindle and is essential for the metaphase to anaphase transition. Cell 81: 261-268.

Vodermaier HC, Gieffers C, Maurer-Stroh S, Eisenhaber F, et al. (2003). TPR subunits of the anaphase-promoting complex mediate binding to the activator protein CDH1. Current Biol. 13: 1459-1468.

Yerle M, Lahbib-Mansais Y, Goureau A, et al. (1999). Contribution to the comparative map between humans and pigs.

Yerle M, Pinton P, Delcros C, et al. (2001). Generation and characterization of a 12,000-rad radiation hybrid panel for fine mapping in pig. Cytogenetic and Genome Res. 97: 219-228.

Yu H, Peters J-M, King RW, Page AM, et al. (1998). Identification of a cullin homology region in a subunit of the anaphasepromoting complex. Science 279: 1219-1222. 